\title{
Medición de recursos personales socio-cognitivos en organizaciones: Análisis psicométricos en trabajadores argentinos
}

\section{Measurement of Personal Socio-Cognitive Resources in Organizations: Psychome- tric Analysis of Argentine Workers}

Leonardo Adrián Medrano ${ }^{1}$, Alvaro Ortiz ${ }^{1}$, Pablo Ezequiel Flores-Kanter ${ }^{*}{ }^{1}$, Sergio Dominguez-Lara

${ }^{2}$, Silvio Gibelli ${ }^{1}$

1 - Universidad Siglo 21, Córdoba, Argentina.

2 - Universidad de San Martín de Porres, Lima, Perú.

\author{
Introducción \\ Método \\ Resultados \\ Discusión \\ Referencias
}

Recibido: 29/11/2017 Revisado: 04/01/2018 Aceptado: 21/01/2018

\section{Resumen}

La teoría de demandas y recursos laborales (Bakker $\&$ Demerouti, 2007) constituye en la actualidad un paradigma de gran importancia para explicar los procesos de motivación y salud ocupacional. En el presente estudio instrumental, se analizan las propiedades psicométricas de tres escalas validadas previamente en una muestra de estudiantes universitarios. Dichas escalas evalúan recursos personales generales, derivados de la teoría social cognitiva, relevantes al ámbito laboral: Escala de Apoyo Social Percibido, Escala de Expectativas de Resultados y Escala de Progreso en Metas. Para llevar a cabo la investigación, se conformó una muestra de 223 trabajadores argentinos. Se efectuaron análisis de validez de constructo y de criterio. Asimismo, la confiabilidad fue evaluada por medio de los coeficientes alfa y omega. Los resultados obtenidos apoyan la unidimensionalidad de las escalas, las relaciones teóricamente relevantes con las dimensiones del burnout, engagement, emociones positivas y satisfacción laboral, así como los indicadores de confiabilidad apropiados. Se discuten las implicancias prácticas de los hallazgos.

Palabras clave: apoyo social percibido, expectativas de resultados, progreso en metas, validez, confiabilidad

\begin{abstract}
The Job Demands-Resources model (Bakker \& Demerouti, 2007) is currently an important framework in explaining health and motivational-related processes at work. The present study examined the psychometric properties of three scales measuring general personal resources previously validated in a sample of university students derived from the Social Cognitive theory related to the workplace: Scale of Perceived Social Support, Scale of Outcome Expectations and Scale of Goal Progress. Two hundred and twenty-three Argentinian workers participated in the study. Internal structure, criterion validity and reliability (Cronbach's alpha and omega) were assessed. The results support the unidimensionality of the scales and theoretically relevant relations with the dimensions of burnout, engagement, positive emotions and job satisfaction as well as appropriate indicators of reliability. Practical implications of the findings are discussed.
\end{abstract}

Key words: perceived social support, outcomes expectations, progress goals, validity, reliability

\footnotetext{
* Correspondencia a: Pablo Ezequiel Flores-Kanter, ezequielfk@gmail.com

Cómo citar este artículo: Medrano, L. A., Ortiz, A., Flores-Kanter, P. E., Dominguez-Lara, S., \& Gibelli, S. (2018). Medición de recursos personales socio-cognitivos en organizaciones: Análisis psicométricos en trabajadores argentinos. Revista Evaluar, 18(2), 01-16. Recuperado de https://revistas.unc. edu.ar/index.php/revaluar
} 


\section{Introducción}

La teoría de Demandas y Recursos (DR) constituye en la actualidad un paradigma de gran importancia para explicar los procesos de motivación y salud ocupacional, y demuestra ser un modelo efectivo para comprender, explicar y pronosticar el desarrollo del burnout, engagement laboral y rendimiento laboral, entre otros factores (Bakker \& Demerouti, 2007). Desde este modelo las demandas laborales son conceptualizadas como los aspectos físicos, psicológicos, organizacionales y sociales del puesto de trabajo que requieren de un esfuerzo sostenido y que conllevan costos fisiológicos y psicológicos. Cabe señalar que las demandas laborales no son negativas o positivas en sí mismas, sino que pueden suponer un obstáculo si requieren un esfuerzo prolongado o bien si la persona no cuenta con los recursos necesarios para afrontarla. Por su parte, los recursos laborales se refieren a los aspectos (físicos, psicológicos, organizacionales o sociales) que: a) reducen las exigencias del trabajo y los costos asociados, b) son necesarios para la consecución de objetivos y c) estimulan el crecimiento personal (Bakker \& Demerouti, 2007; Bakker, Van Veldhoven, \& Xanthopoulou, 2010).

La interacción entre las demandas y los recursos permite explicar los procesos de salud y motivación de los trabajadores. Debido a que las demandas laborales exigen esfuerzo y consumen los recursos energéticos de los trabajadores, constituyen los principales predictores del agotamiento y los problemas de salud psicosomáticos. Por otra parte los recursos laborales permiten satisfacer las necesidades y aspiraciones de los trabajadores, lo que favorece la satisfacción laboral, la motivación y el engagement (Bakker \& Demerouti, 2013). Más allá de la contribución independiente de las demandas y recursos laborales sobre la salud y motivación de los trabajado- res, no debe caerse en una sobresimplificación del modelo asumiendo a priori que los recursos son "positivos" y las demandas "negativas". Es indispensable considerar la interacción entre los mismos (Figura 1). Por ejemplo, Bakker y Demerouti (2013) observaron que el disfrute de la tarea y el compromiso con la organización eran superiores cuando los empleados enfrentaban retos y tareas estimulantes, además de disponer de recursos para afrontarlas. Por el contrario, los trabajos con bajas demandas laborales pueden tornarse aburridos $\mathrm{y}$, consecuentemente, disminuir el bienestar de los empleados.

En una revisión realizada por Bakker y Demerouti (2013) se establecen diferencias entre recursos laborales y recursos personales. Ambos tendrían un efecto sobre el bienestar y el rendimiento de los trabajadores mediante dos vías: 1) indirectamente, al amortiguar el impacto de las demandas sobre el agotamiento de los trabajadores, y 2) directamente, al impactar sobre la motivación y el engagement de los trabajadores. Los recursos personales han sido definidos en términos amplios como autoevaluaciones positivas referidas a la percepción de la capacidad propia de controlar e influir en el entorno. Se ha observado que estas autoevaluaciones predicen el establecimiento de metas, la motivación, el rendimiento laboral y la satisfacción de vida. A mayores recursos personales, más positiva será la autoestima del individuo y mayor concordancia habrá con sus objetivos personales.

En un trabajo realizado por Xanthopoulou, Bakker, Demerouti y Schaufeli (2007) se evaluaron tres recursos personales (autoestima, autoeficacia y optimismo) y se determinó su relación con el engagement y el agotamiento emocional. Los resultados mostraron que los recursos personales no logran anular el efecto de las demandas sobre el agotamiento. Sin embargo, se observó que moderaban de manera significativa 


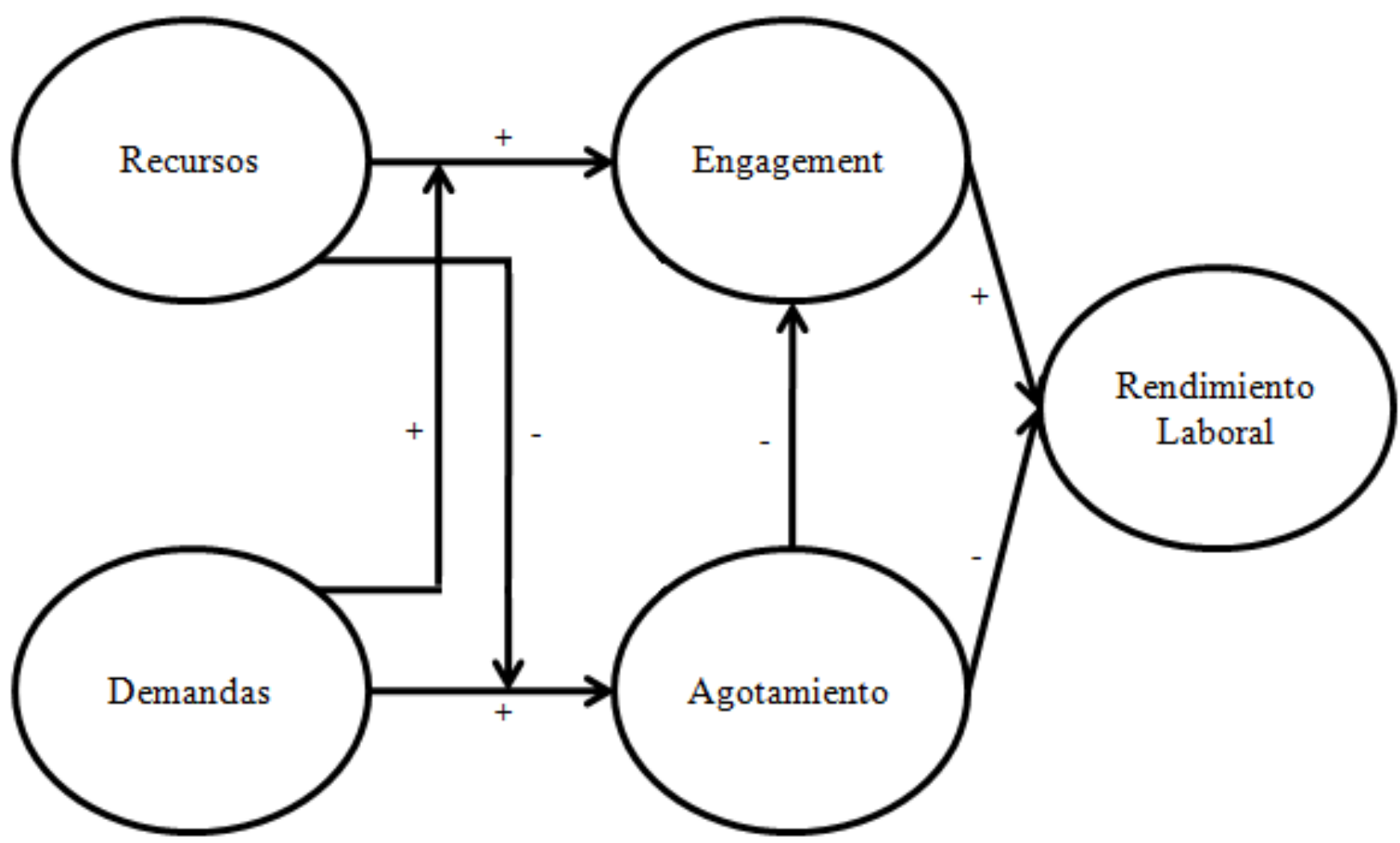

Figura 1

Modelo de Demandas y Recursos (adaptado de Bakker \& Demerouti, 2013)

la relación entre los recursos laborales y el engagement. No obstante, Bakker y Demerouti (2013) señalan que aún es escasa la evidencia sobre la interacción entre los recursos personales y las demandas laborales.

Si bien existen demandas y recursos que son específicos de cada puesto de trabajo, también existen demandas y recursos más generales presentes en cualquier trabajo. En el presente estudio se indaga sobre recursos personales generales derivados de la teoría social cognitiva (TSC; Bandura, 1997) relevantes al ámbito laboral.

\section{Recursos personales socio-cognitivos}

Desde la TSC se postula que las personas no están determinadas por impulsos internos o mol- deadas automáticamente por estímulos externos. Por el contrario, se considera que el comportamiento humano debe analizarse considerando la interacción entre la persona y su ambiente. Desde este enfoque, los seres humanos se caracterizan por poseer un vasto potencial, limitado biológicamente, que puede ser formado por la experiencia directa y observacional. Es decir, se considera que las personas poseen una serie de capacidades básicas que les permiten regular su propia conducta, y configurar a partir de sus acciones las situaciones en que se ven inmersos (Bandura, 1997).

En el transcurrir de su vida diaria, las personas analizan las situaciones que confrontan, consideran diferentes cursos de acción, juzgan sus propias capacidades y evalúan las consecuencias de sus propios actos. De esta manera, logran ejercer un control sobre los numerosos eventos que 
afectan sus vidas. Esta concepción de hombre es sintetizada por Bandura (1997) en el concepto de agencia humana, es decir, que las personas poseen la virtud de obrar sobre sus propias acciones y funcionamiento psicosocial.

En el marco de la TSC, Lent (2008) planteó la existencia de una serie de constructos socio-cognitivos que actuarían como recursos personales en el contexto organizacional y favorecerían la motivación y salud de los trabajadores (Figura 2). El modelo formulado por Lent (2008) subraya el papel del apoyo social percibido, las emociones positivas, las creencias de autoeficacia, las expectativas de resultados y la percepción de progreso en las metas.

En investigaciones previas se ha demostrado el rol de las creencias de autoeficacia y el afecto positivo sobre el engagement de los trabajadores (Castellano, Cifre, Spontón, Medrano, \& Maffei, 2013). Además, se han desarrollado estudios de carácter psicométrico tendientes a evaluar dichas variables en trabajadores argentinos (Ma- ffei, Spontón, Spontón, Castellano, \& Medrano, 2012; Moriondo, De Palma, Medrano, \& Murillo, 2012). Sin embargo, el apoyo social percibido, las expectativas de resultados y el progreso en metas son constructos poco examinados en la literatura científica y de pobre desarrollo en el contexto argentino.

\section{Apoyo Percibido}

El apoyo percibido forma parte de las variables del modelo que tienen que ver con las condiciones laborales (Duffy \& Lent, 2009), y refiere al grado en que los empleados se sienten apoyados por su ambiente laboral para llevar a cabo los objetivos relacionados al trabajo. Según Duffy y Lent (2009) el grado en que los empleados se perciben apoyados en su trabajo está fuertemente relacionado con la satisfacción laboral que experimentan. En la misma línea, Lent y Brown (2008) indican que el grado de respuesta o apoyo

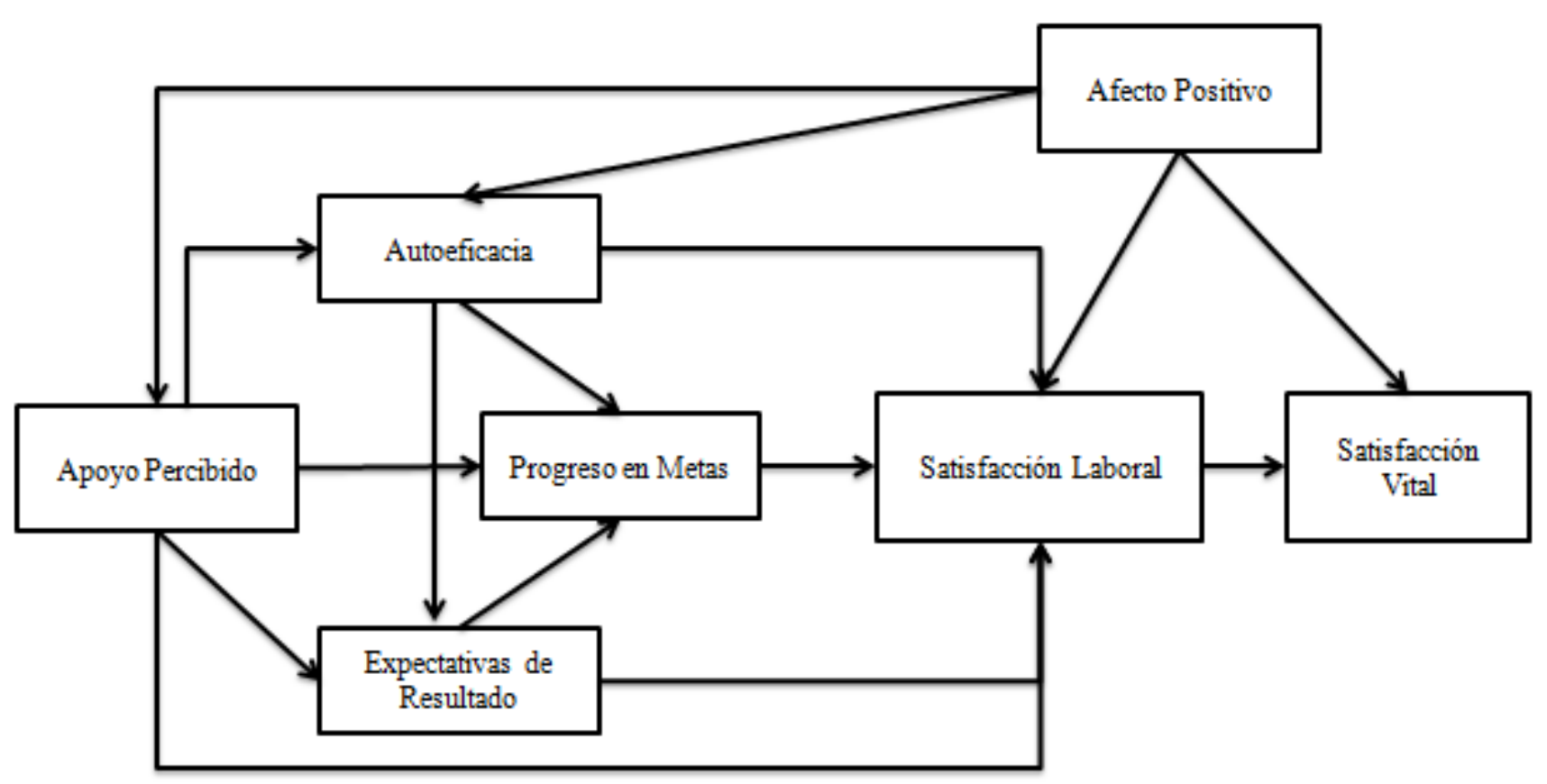

Figura 2

Modelo social cognitivo de satisfacción laboral y satisfacción con la vida (adaptado de Lent, 2004). 
que el empleado percibe en relación con su organización, y el apoyo efectivo que la organización brinda, ofrecen una fuente importante de satisfacción laboral. Al mismo tiempo, se plantea que la falta de apoyo o la presencia de obstáculos que impiden la consecución de las metas de trabajo influyen negativamente sobre la satisfacción laboral (Lent \& Brown, 2006).

Sumado a lo anterior, desde el modelo de Lent (2008) el apoyo percibido no solo afecta a la satisfacción de manera directa, sino también indirectamente por medio de la percepción de progreso en metas, las expectativas de resultados, y las creencias de autoeficacia (Lent \& Brown, 2006). Es importante mencionar también que el apoyo percibido del ambiente organizacional facilita $o$ promueve especialmente el progreso en metas $\mathrm{y}$ la autoeficacia.

Diversas investigaciones han dado cuenta de la relevancia del apoyo organizacional percibido en distintas variables relacionadas con el trabajo. La investigación de Uppal (2017) propone que la percepción de apoyo organizacional actuaría como un recurso en las personas neuróticas promoviendo y facilitando un mejor desempeño laboral, así como un afrontamiento adecuado de situaciones estresantes. Los resultados obtenidos apoyaron estas conjeturas. Se verificó en primer lugar una relación curvilínea entre el neuroticismo y el desempeño laboral, y posteriormente se constató que en los individuos con baja percepción de apoyo, el umbral en el que la relación entre el neuroticismo y el desempeño laboral deja de ser positivo es más bajo . Esto significa que menores niveles de neuroticismo son suficientes para provocar un bajo desempeño laboral en personas con baja percepción de apoyo. Así, los resultados indican que el apoyo percibido modera la relación entre el neuroticismo y el desempeño laboral.

Por su parte, Vatankhah, Javid y Raoofi (2017) estudiaron el efecto mediador del apo- yo organizacional percibido en la relación entre las prácticas para un alto desempeño laboral ( $p$. ej., prácticas para mejorar el conocimiento y las habilidades, oportunidades de promoción, reforzamientos positivos) y las conductas laborales contraproducentes (p. ej., robar, ausentarse). Se hipotetiza que la presencia de estas prácticas aumenta la percepción de apoyo organizacional, lo que propicia una mejor calidad de servicio y menor cantidad de conductas contraproducentes. Los resultados obtenidos van en esta dirección: por un lado se verifica que un incremento en las prácticas de empoderamiento, reforzamiento y promoción aumenta la percepción de apoyo organizacional; por otro lado, el aumento en la percepción de apoyo disminuye las conductas laborales contraproducentes. Por lo tanto se encuentra evidencia a favor de que la percepción de apoyo organizacional media la relación entre las prácticas para un alto desempeño y las conductas contraproducentes.

En un estudio realizado por Cheng, Chen, Teng y Yen (2016), se puso a prueba un modelo en en que el apoyo organizacional percibido moderaba la relación entre la forma de elaboración o invención en el trabajo (i.e. job crafting) con variables laborales como la satisfacción, el compromiso organizacional y el desempeño laboral. Los autores pudieron verificar que en aquellas personas en las que el nivel de soporte percibido era mayor, las relaciones entre el job crafting individual y los resultados laborales eran más fuertes y estadísticamente significativas. Los autores indican que cuando los trabajadores sienten que tienen acceso a recursos adecuados como el apoyo organizacional se mantienen más comprometidos con su trabajo y perciben las demandas como menos intimidantes.

En la misma línea, Caesens y Stinglhamber (2014) indagaron la relación entre la percepción del apoyo organizacional y el engagement laboral, considerando el rol de la autoeficacia. Si bien 
el objetivo central de este trabajo fue verificar el rol mediador de la autoeficacia en la relación entre la percepción de apoyo y el engagement; también se constataron los efectos de esta relación en la satisfacción laboral, la tensión psicológica y el desempeño intra y extra rol. Los resultados indican que el apoyo organizacional percibido tiene un efecto directo y positivo sobre el engagement y la satisfacción laboral; y negativo sobre la tensión psicológica. También se constata su efecto indirecto sobre la satisfacción y la tensión mediante las creencias de autoeficacia y el engagement.

Islam et al. (2013), plantean que el apoyo organizacional juega un rol central en el aumento del compromiso y la retención laboral de empleados con habilidades y actitudes positivas. El modelo que ponen a prueba estos autores indica que el apoyo organizacional percibido tiene un efecto directo sobre la intención de abandono, pero también indirecto sobre esta misma variable a través de su efecto sobre el compromiso organizacional. El modelo propuesto muestra índices de ajuste adecuados, y prueba que el apoyo organizacional percibido tiene un efecto negativo y directo sobre la intención de abandono e indirecto, y positivo sobre el compromiso organizacional, es decir que tiene un efecto negativo sobre la intención de abandono.

Finalmente, Riggle, Edmondson y Hansen (2009) llevaron a cabo un meta-análisis para evaluar el efecto de la percepción de apoyo organizacional sobre el compromiso organizacional, la satisfacción laboral, el desempeño y la intención de abandono. Los resultados obtenidos indican que la percepción de apoyo organizacional tiene un efecto positivo y fuerte sobre la satisfacción laboral y el compromiso organizacional, así como un efecto negativo y fuerte en la intención de abandono. Asimismo, se encuentra un efecto moderado y positivo sobre el desempeño de los empleados.

\section{Expectativas de Resultados}

Más allá de los reforzamientos que los empleados puedan recibir en su entorno laboral, es de importancia también considerar las condiciones laborales o refuerzos que los empleados anticipan que podrán recibir o que serán provistos por su entorno laboral (Lent \& Brown, 2006). En este sentido, Lent y Brown (2006) plantean la relevancia de indagar las creencias de expectativas conceptualizadas como expectativas de resultado, es decir, las creencias acerca de la probabilidad de obtener un particular resultado del trabajo (p. ej., Mi trabajo me permitirá obtener un mejor salario). Se ha demostrado que estas expectativas de resultado explican la satisfacción laboral de los trabajadores.

Además de la satisfacción laboral, las expectativas de resultado han demostrado estar relacionadas con otras variables relevantes en el contexto laboral. Así, Singh et al. (2013) proponen un modelo en el que la autoeficacia y las expectativas de resultado median la relación entre el apoyo social y actitudes laborales como la satisfacción y el compromiso organizacional, lo cual a su vez afecta la decisión de abandonar la empresa. En primer lugar, las correlaciones obtenidas apoyan la hipótesis propuesta dado que las expectativas de resultado correlacionan positivamente con el compromiso organizacional (.38) y la satisfacción laboral (.45) y negativamente con la intención de abandono (-.34). En segundo lugar, el modelo propuesto muestra índices de ajuste adecuados y se puede apreciar que las expectativas de resultado tienen un efecto indirecto sobre la intención de abandono a través de su influencia sobre las actitudes laborales (i.e. satisfacción y compromiso).

Wöhrmann, Deller y Wang (2013), por su parte, indagaron el papel de las expectativas de resultado en las intenciones de continuar trabajando luego del tiempo de retiro. Con base en la 
Teoría Social Cognitiva de la Carrera, el objetivo principal fue indagar cómo las expectativas acerca del trabajo posterior al retiro influyen en las intenciones o planificaciones de seguir trabajando en esa etapa. Los resultados obtenidos permiten ver que las expectativas de resultado tienen un efecto directo y positivo tanto sobre las intenciones de seguir trabajando para el mismo empleador luego del retiro, como sobre las planificaciones para involucrarse en esa etapa en actividades laborales.

\section{Progreso en Metas}

Para la TSC, las metas quedan definidas por la determinación personal para lograr un determinado resultado o alcanzar cierto nivel de dominio (Lent \& Brown, 2006). Asimismo, las metas no solo son un aspecto fundamental para explicar la motivación y el comportamiento de las personas, sino también la satisfacción laboral. Según el modelo propuesto, las metas y las expectativas de resultados poseen un rol central en la explicación de la satisfacción. En este sentido, Lent (2004) señala que las expectativas de resultados afectan la percepción del progreso en metas, y ambas variables influyen directamente sobre los juicios de satisfacción laboral. Esta relación entre las expectativas de resultados y el progreso en metas aparece en función del hecho de que las personas constantemente anticipan las consecuencias de sus acciones, lo que afecta el establecimiento de metas y, finalmente, su ejecución (Lent \& Brown, 2006). En un estudio llevado a cabo por Van Dierendonck (2015) se plantea que la relación entre el progreso en metas y la satisfacción laboral es bidireccional, dado que se espera que las personas con altos niveles de satisfacción se esfuercen en lograr más progreso en sus metas. Al mismo tiempo, el progreso en metas resultaría en la ganancia de mayores recursos y sentimientos positivos, lo que daría lugar a una mayor satisfacción laboral. Los resultados obtenidos apoyan dichas hipótesis; se observa, por un lado, que las medidas de satisfacción en el Tiempo 1 influyen sobre las medidas de progreso en metas en el Tiempo 2, y, por otro lado, que el progreso en metas produce también cambios en la satisfacción desde el Tiempo 1 al Tiempo 2.

Por su parte, Hülsheger y Maier (2010), dan cuenta de la relación entre el progreso en metas y las actitudes laborales, como la satisfacción laboral y el compromiso organizacional. En su estudio concretamente verifican que el nivel de rasgos de estado consciente, es decir, el grado en que la persona está orientada a metas, se focaliza en los logros, y modera la relación entre el progreso en metas, la satisfacción y el compromiso organizacional. Específicamente, los resultados indican que el progreso en metas predice un aumento en la satisfacción y el compromiso organizacional en aquellos casos en los que el estado de consciencia es bajo.

Hyvönen, Feldt, Salmela-Aro, Kinnunen y Mäkikangas (2009) dan cuenta de la relación entre el progreso en metas y variables importantes para el bienestar de los trabajadores como el burnout y el engagement. En términos generales la percepción de progreso en metas se relaciona negativamente con el burnout y sus dimensiones (a excepción del cinismo), y positivamente con el engagement y sus dimensiones. Concretamente, las correlaciones fueron de - .24 con el burnout, de -.11 con el cansancio emocional y de - .26 con la reducción de la eficacia personal. Por su parte, las correlaciones con el engagement fueron de .30, con el vigor de .29 , con la dedicación de .33 y con la absorción de .18. A partir de los resultados se obtuvo evidencia de que a mayor percepción de progreso en metas es más probable que los empleados experimenten engagement y menos probable que manifiesten burnout. 
Maier y Brunstein (2001) plantean finalmente que es más probable que los empleados logren niveles altos de satisfacción y compromiso organizacional si perciben que su empresa promueve el alcance de metas valoradas. En su estudio los autores encuentran evidencia a favor de la hipótesis que plantea que el progreso en metas presenta un rol intermediario en la predicción de la satisfacción y el compromiso organizacional. Más específicamente, la presencia de un alto compromiso por las metas así como condiciones favorables para su consecución da lugar a una mayor percepción de progreso en metas lo que culmina en una mayor satisfacción laboral y compromiso organizacional.

\section{Objetivos de la presente investigación}

Dado el hecho de que no se cuenta con instrumentos debidamente adaptados que permitan una medición de los recursos socio-cognitivos y que presenten evidencias de validez y confiabilidad, el presente trabajo tiene por objetivo principal evaluar las propiedades psicométricas de tres instrumentos que posibilitan medir dichos recursos: la Escala de Apoyo Social Percibido (Lent et al., 2005), la Escala de Expectativas de Resultados (Lent et al., 2003) y la Escala de Progreso en Metas (Lent et al., 2005). Esos instrumentos han mostrado indicadores psicométricos favorables en estudios previos, aunque llevados a cabo con estudiantes universitarios (Medrano, Pérez, \& Fernandez-Liporace, 2014). Sin embargo, es razonable pensar que al basarse en la TSC, los resultados sean consistentes en una población adulta no universitaria. Tomando como base las escalas validadas con estudiantes universitarios, esta investigación buscará ampliar su validación con una muestra de trabajadores argentinos.
Las estrategias para obtener evidencias de validez se enmarcarán en el análisis de la estructura interna de los instrumentos, así como en su relación con otras variables (burnout, engagement y emociones positivas). Contar con esos instrumentos validados facilitaría la evaluación del modelo teórico de Lent (2008) en trabajadores argentinos y de ese modo promovería acciones orientadas a la mejora de las condiciones laborales desde el quehacer del psicólogo.

\section{Método \\ Participantes}

Se contó con la participación de 223 trabajadores seleccionados mediante muestreo accidental no probabilístico. El 56\% de la muestra contestó los cuestionarios de manera presencial, mientras que el restante $44 \%$ lo hizo vía e-mail. En este último caso se les remitió a los participantes la encuesta vía correo electrónico y en formato Word, una vez contestada esta debía ser reenviada a los investigadores. La muestra estuvo compuesta por varones (63\%) y mujeres $(34 \%)$ con edades de entre los 19 y 67 años $(\mathrm{M}=34.7$; $\mathrm{DE}=9.35)$. Con el fin de contar con una muestra heterogénea, se incluyeron trabajadores de diferentes áreas y puestos (Administración $=10.2 \%$; Atención al cliente $=3 \%$; Comercio $=7 \%$; Ventas $=6 \%$, entre otros). La antigüedad laboral promedio fue de 4.11 años y el promedio de jornada laboral fue de 41.42 horas semanales. Finalmente, resta señalar que el tamaño muestral utilizado resulta adecuado para efectuar los análisis estadísticos planificados para el estudio (Lloret-Segura, Ferreres-Traver, Hernández-Baeza, \& Tomás-Marco, 2014). 


\section{Instrumentos}

Escala de Apoyo Percibido. El instrumento se compone de nueve ítems que permiten evaluar en qué medida el contexto próximo al trabajador lo apoya en la consecución de sus objetivos laborales (p. ej., Siento que mis compañeros me alientan para alcanzar mis objetivos laborales). Para responder los participantes deben señalar su nivel de acuerdo con cada afirmación utilizando una escala tipo Likert con ítems que van desde $M u y$ en desacuerdo (1) hasta Totalmente de acuerdo (10). Los estudios psicométricos previos indican una consistencia interna satisfactoria ( $\alpha$ entre .77 y .84) y una estructura unidimensional tanto en la muestra de trabajadores (Lent et al., 2007) como en la de estudiantes universitarios (Medrano et al., 2014).

Escala de Expectativas de Resultados. Se compone de diez ítems que miden las consecuencias que perciben los trabajadores de su trabajo. Para ello se inicia con la consigna: Esta experiencia laboral probablemente me permita: , seguido de 10 ítems (p. ej., Ganar un mejor salario; Aumentar mi autoestima). Para responder, los participantes deben indicar su grado de acuerdo utilizando una escala Likert de 10 posiciones (desde Estoy en fuerte desacuerdo; hasta Estoy fuertemente de acuerdo). Los estudios psicométricos previos llevados a cabo tanto en estudiantes como en trabajadores señalan que este instrumento presenta una óptima y adecuada consistencia interna ( $\alpha$ entre .72 y .91) y una estructura unidimensional (Lent et al., 2007; Medrano et al., 2014).

Escala de Progreso en Metas. El instrumento se compone de seis ítems que evalúan el progreso que los trabajadores perciben en sus metas. En el mismo los participantes deben indicar en qué medida han superado cada una de las metas planteadas en los reactivos (p. ej., Destacarme en mi trabajo; Completar exitosamente mis tareas), uti- lizando 10 opciones de respuesta (desde No he progresado del todo hasta He realizado un excelente progreso). Los estudios psicométricos previos dan cuenta de una estructura unidimensional y una adecuada consistencia interna ( $\alpha$ entre $.81 \mathrm{y}$ .89; Lent et al., 2007; Medrano et al., 2014).

Medición del Afecto Positivo. Se utilizó la subescala de afecto positivo de la Escala de Afecto Positivo y Negativo (PANAS). La misma fue adaptada en la población local por Moriondo et al. (2012). Los resultados de los análisis psicométricos sugieren una estructura de dos dimensiones (afecto positivo y negativo) y niveles óptimos de confiabilidad (afecto positivo $\alpha=.73$; afecto negativo $\alpha=.82$, respectivamente). La dimensión de afecto positivo cuenta de 10 ítems que describen distintas emociones positivas (p. ej., Fuerte, Inspirado), en los que la persona debe indicar con qué frecuencia experimenta cada una de ellas utilizando una escala de cinco posiciones.

Maslach Burnout Inventory-General Survey (MBI-GS). Fue usada la versión adaptada al medio local por Spontón, Maffei, Spontón, Medrano y Castellano (2011). La escala permite medir tres factores subyacentes, consistentes con los propuestos en estudios previos: 1) agotamiento (4 ítems; p. ej., Me encuentro agotado al final de la jornada laboral), 2) despersonalización (4 ítems; p. ej., Trato a algunas personas de mi trabajo como si fueran objetos) y 3) cinismo (5 ítems; p. ej., He perdido interés y entusiasmo en este trabajo). Para responder a los ítems, los examinados deben utilizar una escala de respuesta de siete puntos que va desde 0 (Nunca) hasta 6 (Siempre). Los estudios de consistencia interna de la escala revelaron niveles aceptables de homogeneidad entre los ítems $(\alpha>$.70).

Utrecht Work Engagement Scale (UWES). Fue aplicada la adaptación realizada en Córdoba, Argentina, por Spontón, Medrano, Maffei, Spontón y Castellano (2012). El UWES contiene 17 ítems 
distribuidos en tres factores correspondientes a las dimensiones teóricas del engagement: 1) vigor (6 ítems; p. ej., En mi trabajo me siento lleno de energía), 2) dedicación (6 ítems; p. ej., Mi trabajo tiene sentido) y 3) absorción (5 ítem; p. ej., Cuando estoy trabajando olvido todo lo que pasa a mi alrededor) y un formato de respuesta Likert de siete posiciones que va desde 0 (Nunca) hasta 6 (Siempre). Los análisis psicométricos de la escala en la población de trabajadores cordobeses evidencian un agrupamiento de los ítems en tres factores distintos, lo que sugiere una estructura interna de tres factores compatibles con las dimensiones teóricas del engagement. Los estudios de confiabilidad de la escala indican que las dimensiones poseen niveles adecuados $(\alpha=.88$ para dedicación; $\alpha=.76$ para vigor; $\mathrm{y} \alpha=.69$ para absorción).

\section{Procedimiento y Análisis de datos}

En la presente investigación se llevó a cabo un estudio de carácter instrumental (Montero \& León, 2007). Para ello se administraron los instrumentos mencionados de manera presencial y virtual, se les comunicó a los trabajadores el carácter voluntario de su participación y se les garantizó la confidencialidad de sus respuestas.

Respecto al análisis de datos, para el análisis factorial exploratorio (AFE) se tuvieron en cuenta las sugerencias dadas por la literatura especializada (Ferrando \& Lorenzo-Seva, 2014; Lloret-Segura et al., 2014). Se consideraron así para el análisis, a) la matriz de correlaciones producto-momento de Pearson, b) el análisis paralelo (Horn, 1965) para la determinación del número de factores y c) el método de estimación de mínimos cuadrados no ponderados (i. e. ULS). Este último método se muestra robusto y logra estimaciones más precisas aun cuando se incumplen los supues- tos de normalidad en los ítems. No fueron implementados procedimientos de rotación factorial debido a que se trata de cuestionarios unidimensionales. Finalmente, en cuanto a las evidencias de validez, se obtuvieron resultados adicionales respecto a su relación con variables externas teóricamente relevantes (validez de criterio). Para todos estos análisis se utilizó el software FACTOR (Ferrando \& Lorenzo-Seva, 2014) para el análisis factorial y el SPSS v.20 (IBM Corp., 2011) para las correlaciones con los criterios externos.

En cuanto al análisis de la confiabilidad, fueron empleados el coeficiente $\omega$ (McDonald, 1999) y el coeficiente $\alpha$ desde el modelo del factor común (Dominguez-Lara, 2012), dado que en ausencia del cumplimiento del supuesto de tau-equivalencia (Dominguez-Lara, 2016) no pudo usarse el coeficiente $\alpha$ (Cronbach, 1951).

\section{Resultados}

Análisis exploratorios iniciales de los ítems

En primer lugar, fueron analizados los estadísticos descriptivos de los ítems de cada cuestionario. En general, se aprecia un predominio de las respuestas en el polo elevado de la escala y, aunque la asimetría y la curtosis se mantienen en magnitudes adecuadas, los últimos ítems de la Escala de Progreso en Metas poseen estadísticos de distribución elevados (Tabla 1).

\section{Tabla 1}

Recursos sociocognitivos laborales: Análisis descriptivo de los ítems.

\begin{tabular}{ccccc}
\hline \multicolumn{5}{c}{ Expectativa de Resultado } \\
\hline Ítems & $\mathrm{M}$ & $\mathrm{DE}$ & $\mathrm{g} 1$ & $\mathrm{~g} 2$ \\
1 & 7.25 & 2.12 & -.80 & .57 \\
2 & 7.00 & 2.25 & -.72 & .36 \\
\hline
\end{tabular}




\begin{tabular}{|c|c|c|c|c|}
\hline 3 & 7.00 & 2.15 & -.83 & .68 \\
\hline 4 & 7.78 & 2.00 & -1.15 & 1.67 \\
\hline 5 & 7.13 & 2.28 & -.76 & .25 \\
\hline 6 & 6.46 & 2.45 & -.47 & -.28 \\
\hline 7 & 7.13 & 2.44 & -.89 & .23 \\
\hline 8 & 6.67 & 2.47 & -.74 & -.06 \\
\hline 9 & 7.21 & 2.37 & -.88 & .22 \\
\hline 10 & 7.82 & 1.99 & -1.17 & 1.38 \\
\hline \multicolumn{5}{|c|}{ Apoyo Percibido } \\
\hline & M & $\mathrm{DE}$ & g1 & g2 \\
\hline 1 & 6.13 & 2.61 & -.39 & -.65 \\
\hline 2 & 7.04 & 2.26 & -.81 & .20 \\
\hline 3 & 7.04 & 2.34 & -.75 & .11 \\
\hline 4 & 6.66 & 2.92 & -.68 & -.67 \\
\hline 5 & 6.63 & 2.38 & -.59 & -.33 \\
\hline 6 & 6.61 & 2.53 & -.60 & -.36 \\
\hline 7 & 8.22 & 2.08 & -1.29 & 1.23 \\
\hline 8 & 7.50 & 2.14 & -.70 & -.01 \\
\hline 9 & 6.64 & 2.75 & -.64 & -.61 \\
\hline \multicolumn{5}{|c|}{ Progreso en Metas } \\
\hline & M & $\mathrm{DE}$ & g1 & $\mathrm{g} 2$ \\
\hline 1 & 7.42 & 1.54 & -.37 & -.29 \\
\hline 2 & 8.10 & 1.35 & -.63 & .38 \\
\hline 3 & 8.35 & 1.32 & -.98 & .83 \\
\hline 4 & 7.26 & 2.62 & -.77 & -.28 \\
\hline 5 & 8.37 & 1.35 & -1.49 & 4.22 \\
\hline 6 & 7.61 & 1.67 & -1.09 & 2.15 \\
\hline 7 & 8.66 & 1.44 & -1.90 & 6.23 \\
\hline
\end{tabular}

Nota. $\mathrm{N}=223$; M: Media; DE: Desviación Estándar; g1: asimetría; g2: curtosis

\section{Análisis factorial confirmatorio}

Posteriormente, previo al AFE, fue verificada la adecuación de la matriz de correlaciones. Los resultados indican que la matriz es adecuada tanto para los análisis de la Escala de Expectativas de Resultado $(\chi 2=615.2, p<.001 ; \mathrm{KMO}=$ .83) como de la Escala de Apoyo Percibido $(\chi 2$ $=870.8, p<.001 ; \mathrm{KMO}=.81)$ y de la Escala de Progreso en Metas $(\chi 2=615.2, p<.001 ; \mathrm{KMO}$
$=.83)$. En todos los casos, el análisis paralelo indicó que es necesaria la extracción de un solo factor. En la Tabla 2 se sintetizan los resultados encontrados.

Tabla 2

Recursos sociocognitivos laborales: Cargas factoriales, comunalidad, varianza explicada, y consistencia interna.

\begin{tabular}{|c|c|c|c|c|c|c|}
\hline \multirow[b]{2}{*}{ Ítems } & \multicolumn{2}{|c|}{$\begin{array}{c}\text { Expectativa } \\
\text { de Resultado }\end{array}$} & \multicolumn{2}{|c|}{$\begin{array}{c}\text { Apoyo } \\
\text { Percibido }\end{array}$} & \multicolumn{2}{|c|}{$\begin{array}{c}\text { Progreso en } \\
\text { Metas }\end{array}$} \\
\hline & $\lambda$ & $\mathrm{h} 2$ & $\lambda$ & $\mathrm{h} 2$ & $\lambda$ & h2 \\
\hline 1 & .67 & .45 & .58 & .34 & .65 & .43 \\
\hline 2 & .61 & .37 & .72 & .51 & .72 & .52 \\
\hline 3 & .77 & .60 & .48 & .23 & .76 & .58 \\
\hline 4 & .80 & .64 & .65 & .43 & .45 & .20 \\
\hline 5 & .74 & .55 & .70 & .49 & .74 & .56 \\
\hline 6 & .58 & .33 & .77 & .60 & .68 & .46 \\
\hline 7 & .57 & .33 & .37 & .14 & .65 & .43 \\
\hline 8 & .62 & .39 & .37 & .14 & & \\
\hline 9 & .65 & .43 & .79 & .63 & & \\
\hline 10 & .63 & .40 & & & & \\
\hline $\begin{array}{l}\text { Varianza } \\
\text { Explicada }\end{array}$ & .51 & & .45 & & .53 & \\
\hline$\alpha$ & .89 & & .84 & & .85 & \\
\hline$\omega$ & .89 & & .84 & & .85 & \\
\hline
\end{tabular}

Nota. $\mathrm{N}=223 ; \lambda$ : carga factorial; $\mathrm{h} 2$ : comunalidad; $\alpha$ : coeficiente alfa desde el modelo del factor común; $\omega$ : coeficiente omega.

Para todas las escalas se verifican cargas factoriales adecuadas $(>.30)$, así como una consistencia interna óptima $(>.80)$.

\section{Análisis de homogeneidad de los items}

Complementariamente, se analizó la homogeneidad de los ítems mediante la correlación 
ítem-total corregida. Los resultados se exponen en la Tabla 3.

Tabla 3

Correlación Ítem-Total (I-T) y coeficiente $\alpha$ si se elimina el elemento.

\begin{tabular}{|c|c|c|c|c|c|c|}
\hline \multirow[b]{2}{*}{ Ítems } & \multicolumn{2}{|c|}{$\begin{array}{c}\text { Expectativa de } \\
\text { Resultado }\end{array}$} & \multicolumn{2}{|c|}{$\begin{array}{c}\text { Apoyo } \\
\text { Percibido }\end{array}$} & \multicolumn{2}{|c|}{$\begin{array}{l}\text { Progreso en } \\
\text { Metas }\end{array}$} \\
\hline & $\begin{array}{l}\text { Correla- } \\
\text { ción I-T }\end{array}$ & $\begin{array}{l}\alpha \text { si se } \\
\text { elimina } \\
\text { el ele- } \\
\text { mento }\end{array}$ & $\begin{array}{c}\text { Corre- } \\
\text { lación } \\
\text { I-T }\end{array}$ & $\begin{array}{c}\alpha \text { si } \\
\text { se } \\
\text { elimi- } \\
\text { na el } \\
\text { ele- } \\
\text { men- } \\
\text { to }\end{array}$ & $\begin{array}{l}\text { Co- } \\
\text { rrela- } \\
\text { ción } \\
\text { I-T }\end{array}$ & $\begin{array}{l}\alpha \text { si se } \\
\text { elimina } \\
\text { el ele- } \\
\text { mento }\end{array}$ \\
\hline 1 & .63 & .87 & .53 & .83 & .59 & .78 \\
\hline 2 & .57 & .88 & .66 & .81 & .61 & .78 \\
\hline 3 & .71 & .87 & .44 & .83 & .64 & .78 \\
\hline 4 & .74 & .86 & .57 & .82 & .42 & .85 \\
\hline 5 & .69 & .87 & .64 & .81 & .66 & .78 \\
\hline 6 & .54 & .88 & .70 & .81 & .63 & .78 \\
\hline 7 & .55 & .88 & .36 & .84 & .58 & .79 \\
\hline 8 & .59 & .87 & .35 & .84 & & \\
\hline 9 & .61 & .87 & .72 & .80 & & \\
\hline 10 & .60 & .87 & & & & \\
\hline
\end{tabular}

Análisis de validez de criterio

Finalmente, se evaluó la validez de criterio de los recursos sociocognitivos laborales mediante su relación con las dimensiones del burnout y del engagement, así como con las emociones positivas. Como puede apreciarse en la Tabla 4, fueron halladas correlaciones significativas y positivas entre los recursos sociocognitivos laborales por una parte, y el engagement y las emociones positivas por otra. Por el contrario, las correlaciones fueron negativas con el burnout. La mayoría de las correlaciones presentaron, además de significancia estadística, significancia práctica
(Fritz, Morris, \& Richler 2012).

\section{Tabla 4}

Correlaciones bivariadas entre los factores de recursos sociocognitivos laborales estimados con los niveles de burnout, engagement, emociones positivas y satisfacción laboral.

\begin{tabular}{lccc}
\hline & $\begin{array}{c}\text { Progreso } \\
\text { en Metas }\end{array}$ & $\begin{array}{l}\text { Apoyo } \\
\text { Percibido }\end{array}$ & $\begin{array}{c}\text { Expectativas } \\
\text { de Resultado }\end{array}$ \\
\hline $\begin{array}{c}\text { Emociones } \\
\text { Positivas }\end{array}$ & $.37 * *$ & $.41^{* *}$ & $.57 * *$ \\
Agotamiento & $-.16^{*}$ & $-.31 * *$ & -.12 \\
Cinismo & -.05 & $-.25 * *$ & .01 \\
Despersonali- & $-.20^{* *}$ & $-.39 * *$ & $-.42 * *$ \\
zación & & & $-.24 * *$ \\
Dedicación & -.06 & $-.24 * *$ \\
Absorción & $.22 * *$ & $.44 * *$ & $.53 * *$ \\
Vigor & $.21 * *$ & $.38^{* *}$ & $.49 * *$ \\
\hline Nota. ${ }^{*} p<.05 ; * * p<.01$ & &
\end{tabular}

\section{Discusión}

En el marco de la TSC, Lent (2008) planteó la existencia de una serie de constructos sociocognitivos que actuarían como recursos personales en el contexto organizacional y favorecerían la motivación y la salud de los trabajadores. Específicamente, el modelo formulado subraya el papel del apoyo social percibido, las emociones positivas, las creencias de autoeficacia, las expectativas de resultados y la percepción de progreso en las metas.

El presente proyecto tuvo como objetivo principal evaluar las propiedades psicométricas de la Escala de Apoyo Social Percibido, de la Escala de Expectativas de Resultados Organizacionales y de la Escala de Progreso en Metas Organizacionales en una muestra de trabajadores de Argentina. Los resultados obtenidos apoyan la unidimensionalidad de las escalas, de manera congruente con estudios previos (Lent et al., 
2003, 2005), su relación con los constructos teóricamente relevantes, y ofrecen indicadores de confiabilidad apropiados.

Respecto a la confiabilidad, en los análisis se han observado valores adecuados y óptimos de consistencia interna $(\alpha>$.80). En relación con las evidencias de validez, y en concordancia con el modelo de Lent (2008), los análisis dan cuenta de correlaciones significativas mayoritariamente moderadas y fuertes que indican una varianza compartida entre el 10 y el $38 \%$ con las dimensiones del burnout, el engagement y las emociones positivas. Los resultados obtenidos son coherentes con las hipótesis planteadas; de esta manera, se obtienen evidencias de validez con criterios externos. Desde los postulados iniciales de Bandura (1997), se indica que estos recursos sociocognitivos son una fuente importante de motivación, esfuerzo y perseverancia en las tareas, por lo cual era esperable que estas se relacionaran positivamente con el engagement y negativamente con el burnout. En efecto, los recursos se relacionan positivamente con las percepciones de dedicación, vigor y absorción en las tareas del trabajo ( $r$ entre .215 y .531). De manera opuesta, las variables se relacionan negativamente con el agotamiento, el cinismo y la despersonalización ( $r$ entre -.182 y -.421).

Si bien el tamaño muestral es adecuado, considerando la cantidad de ítems de cada instrumento analizado, se sugiere para futuros estudios ampliar la muestra. Por otro lado, las respuestas se caracterizan por situarse en el polo alto de las posibles respuestas para cada ítem (entre 7 y 10). Ante ello, sería conveniente implementar medidas de deseabilidad social para controlar la potencial impresión positiva que probablemente brinden los evaluados. De igual manera, a pesar de que los datos presentan una distribución asimétrica, se han obtenido adecuadas propiedades psicométricas.

En síntesis, se brindan instrumentos con evidencias de validez y confiabilidad para la medición del apoyo social percibido, las expectativas de resultados y el progreso en metas en el ámbito del trabajo, aunque queda pendiente evaluar sus capacidades predictivas respecto a la satisfacción laboral (Lent et al., 2007). Se espera que este trabajo contribuya a un mayor desarrollo de las investigaciones de estos constructos sociocognitivos en el contexto laboral argentino en vista de su fortaleza conceptual y potencial aplicación en diversas áreas de la psicología.

\section{Referencias}

Bakker, A. B., \& Demerouti, E. (2007). The Job Demands-Resources model: State of the art. Journal of Managerial Psychology, 22(3), 309-328. doi: 10.1108/02683940710733115

Bakker, A. B., \& Demerouti, E. (2013). La teoría de las demandas y los recursos laborales. Journal of Work and Organizational Psychology, 29(3), 107-115. doi: $10.5093 / \operatorname{tr} 2013 \mathrm{a} 16$

Bakker, A. B., Van Veldhoven, M., \& Xanthopoulou, D. (2010). Beyond the Demand-Control model: Thriving on high job demands and resources. Journal of Personnel Psychology, 9(1), 3-16. doi: 10.1027/18665888/a000006

Bandura, A. (1997). Self-efficacy: The exercise of control. New York, NY: W. H. Freeman.

Caesens, G., \& Stinglhamber, F. (2014). The relationship between perceived organizational support and work engagement: The role of self-efficacy and its outcomes. Revue Européenne de Psychologie Appliquée, 64(5), 259-267. doi: 10.1016/j.erap.2014.08.002

Castellano, E., Cifre, E., Spontón, C., Medrano, L. A., \& Maffei, L. (2013). Emociones positivas y negativas en la predicción del burnout y engagement en el trabajo. Revista Peruana de Psicología y Trabajo So- 
cial, 2(1), 75-88. Recuperado de http://repositori.uji. es

Cheng, J. C., Chen, C. Y., Teng, H. Y., \& Yen, C. H. (2016). Tour leaders' job crafting and job outcomes: The moderating role of perceived organizational support. Tourism Management Perspectives, 20, 19-29. doi: 10.1016/j.tmp.2016.06.001

Cronbach, L. J. (1951). Coefficient alpha and the internal structure of tests. Psychometrika, 16(3), 297-334. doi: $10.1007 / \mathrm{bf02310555}$

Dominguez-Lara, S. (2012). Propuesta para el cálculo del Alfa Ordinal y Theta de Armor. Revista de Investigación en Psicología, 15(1), 213-217. doi: 10.15381/ rinvp.v15i1.3684

Dominguez-Lara, S. A. (2016). Secretos del coeficiente alfa. Actas Urológicas Españolas, 40(7), 471. doi: 10.1016/j.acuro.2016.04.002

Duffy, R. D., \& Lent, R. W. (2009). Test of a social cognitive model of work satisfaction in teachers. Journal of Vocational Behavior, 75(2), 212-223. doi: 10.1016/j. jvb.2009.06.001

Ferrando, P. J., \& Lorenzo-Seva, U. (2014). El análisis factorial exploratorio de los ítems: Algunas consideraciones adicionales. Anales de Psicología, 30(3), 1170-1175. doi: 10.6018/analesps.30.3.199991

Fritz, C. O., Morris, P. E., \& Richler, J. J. (2012). Effect size estimates: Current use, calculations, and interpretation. Journal of Experimental Psychology: General, 141(1), 2-18. doi: 10.1037/a0024338

Horn, J. L. (1965). A rationale and test for the number of factors in factor analysis. Psychometrika, 30(2), 179185. doi: 10.1007/bf02289447

Hülsheger, U. R., \& Maier, G. W. (2010). The careless or the conscientious: Who profits most from goal progress? Journal of Vocational Behavior, 77(2), 246254. doi: 10.1016/j.jvb.2010.04.001

Hyvönen, K., Feldt, T., Salmela-Aro, K., Kinnunen, U., \& Mäkikangas, A. (2009). Young managers' drive to thrive: A personal work goal approach to burnout and work engagement. Journal of Vocational Behavior,
75(2), 183-196. doi: 10.1016/j.jvb.2009.04.002

IBM Corp. (2011). IBM SPSS Statistics for Windows, Version 20.0. [software de cómputo]. Armonk, NY: IBM Corp.

Islam, T., Khan, S. ur R., Ungku-Ahmad, U. N. B, Ali, G., Ahmed, I., \& Bowra, Z. A. (2013). Turnover intentions: The influence of perceived organizational support and organizational commitment. Procedia: Social and Behavioral Sciences, 103, 1238-1242. doi: 10.1016/j.sbspro.2013.10.452

Lent, R. W. (2004). Toward a unifying theoretical and practical perspective on well-being and psychosocial adjustment. Journal of Counseling Psychology, 51(4), 482-509. doi: 10.1037/0022-0167.51.4.482

Lent, R. W. (2008). Understanding and promoting work satisfaction. An integrative view. En S. D. Brown \& R. W. Lent (Eds.), Handbook of Counseling Psychology (4 ed., pp. 462-482). New York, NY: Wiley.

Lent, R. W., \& Brown, S. D. (2006). Integrating person and situation perspectives on work satisfaction: A social-cognitive view. Journal of Vocational Behavior, 69(2), 236-247. doi: 10.1016/j.jvb.2006.02.006

Lent, R. W., \& Brown, S. D. (2008). Social cognitive career theory and subjective well-being in the context of work. Journal of Career Assessment, 16(1), 6-21. doi: 10.1177/1069072707305769

Lent, R. W., Brown, S. D., Schmidt, J., Brenner, B., Lyons, H., \& Treistman, D. (2003). Relation of contextual supports and barriers to choice behavior in engineering majors: Test of alternative social cognitive models. Journal of Counseling Psychology, 50(4), 458465. doi: 10.1037/0022-0167.50.4.458

Lent, R. W., Singley, D., Sheu, H. B., Gainor, K. A., Brenner, B. R., Treistman, D., \& Ades, L. (2005). Social cognitive predictors of domain and life satisfaction: Exploring the theoretical precursors of subjective well-being. Journal of Counseling Psychology, 52(3), 429-442. doi: 10.1037/0022-0167.52.3.429

Lent, R. W., Singley, D., Sheu, H. B., Schmidt, J. A., \& 
Schmidt, L. C. (2007). Relation of social-cognitive factors to academic satisfaction in engineering students. Journal of Career Assessment, 15(1), 87-97. doi: 10.1177/1069072706294518

Lloret-Segura, S., Ferreres-Traver, A., Hernández-Baeza, A., \& Tomás-Marco, I. (2014). El análisis factorial exploratorio de los ítems: una guía práctica, revisada y actualizada. Anales de Psicología, 30(3), 11511169. doi: 10.6018/analesps.30.3.199361

Maffei, L., Spontón, C., Spontón, M., Castellano, E., \& Medrano, L. A. (2012). Adaptación del Cuestionario de Autoeficacia Profesional (AU-10) a la población de trabajadores cordobeses. Pensamiento Psicológico, 10(1), 51-62. Recuperado de http://revistas.javerianacali.edu.co/index.php/pensamientopsicologico

Maier, G. W., \& Brunstein, J. C. (2001). The role of personal work goals in newcomers' job satisfaction and organizational commitment: A longitudinal analysis. Journal of Applied Psychology, 86(5), 1034-1042. doi: 10.1037/0021-9010.86.5.1034

McDonald, R. P. (1999). Test theory: A unified treatment. Mahwah, NJ: Lawrence Erlbaum Associates. doi: 10.4324/9781410601087

Medrano, L. A., Pérez, E., \& Fernandez-Liporace, M. (2014). Sistema de Evaluación Informatizado de la Satisfacción Académica para ingresantes a la universidad. Electronic Journal of Research in Educational Psychology, 12(33), 541-562. doi: 10.25115/ ejrep.33.13131

Montero, I., \& León, O. G. (2007). A guide for naming research studies in Psychology. International Journal of Clinical and Health Psychology, 7(3), 847-862. Recuperado de http://www.aepc.es/ijchp/

Moriondo, M., De Palma, P., Medrano, L. A., \& Murillo, P. (2012). Adaptación de la Escala de Afectividad Positiva y Negativa (PANAS) a la población de adultos de la ciudad de Córdoba: Análisis psicométricos preliminares. Universitas Psychologica, 11(1), 187-196. Recuperado de http://www.redalyc.org

Riggle, R. J., Edmondson, D. R., \& Hansen, J. D. (2009). A meta-analysis of the relationship between perceived organizational support and job outcomes: 20 years of research. Journal of Business Research, 62(10), 1027-1030. doi: 10.1016/j.jbusres.2008.05.003

Singh, R., Fouad, N. A., Fitzpatrick, M. E., Liu, J. P., Cappaert, K. J., \& Figuereido, C. (2013). Stemming the tide: Predicting women engineers' intentions to leave. Journal of Vocational Behavior, 83(3), 281-294. doi: 10.1016/j.jvb.2013.05.007

Spontón, C., Maffei, L., Spontón, M., Medrano, L. A., \& Castellano, E. (2011). Adaptación psicométrica del cuestionario de burnout MBI-GS a la población de trabajadores de Córdoba, Argentina. Manuscrito inédito.

Spontón, C., Medrano, L. A., Maffei, L., Spontón, M., \& Castellano, E. (2012). Validación psicométrica del cuestionario de engagement UWES a la población de trabajadores de Córdoba, Argentina. Liberabit, 18(2), 147-154. Recuperado de http://revistaliberabit.com/es/

Uppal, N. (2017). Moderation effects of perceived organisational support on curvilinear relationship between neuroticism and job performance. Personality and Individual Differences, 105, 47-53. doi: 10.1016/j. paid.2016.09.030

Van Dierendonck, D. (2015). The influence of planning, support and self-concordance on goal progress and job satisfaction. Evidence-based HRM: a Global Forum for Empirical Scholarship, 3(3), 206-221. doi: 10.1108/EBHRM-04-2014-0013

Vatankhah, S., Javid, E., \& Raoofi, A. (2017). Perceived organizational support as the mediator of the relationships between high-performance work practices and counter-productive work behavior: Evidence from airline industry. Journal of Air Transport Management, 59, 107-115. doi: 10.1016/j.jairtraman.2016.12.001

Wöhrmann, A. M., Deller, J., \& Wang, M. (2013). Outcome expectations and work design characteristics in post-retirement work planning. Journal of Vo- 
cational Behavior, 83(3), 219-228. doi: 10.1016/j. jvb.2013.05.003

Xanthopoulou, D., Bakker, A. B., Demerouti, E., \& Schaufeli, W. B. (2007). The role of personal resources in the job demands-resources model. International Journal of Stress Management, 14(2), 121-141. doi: 10.1037/1072-5245.14.2.121 